\title{
REVIEW
}

\section{Secondary health conditions in individuals aging with SCl: Terminology, concepts and analytic approaches}

\author{
MP Jensen ${ }^{1}$, IR Molton ${ }^{1}$, SL Groah ${ }^{2}$, ML Campbell $^{3}$, S Charlifue ${ }^{4}$, A Chiodo $^{5}$, M Forchheimer $^{5}$, JS Krause ${ }^{6}$ \\ and D Tate ${ }^{5}$
}

\section{Study design: Literature review.}

Objectives: Utilizing individuals with spinal cord injury ( $\mathrm{SCl}$ ) as a representative population for physical disability, this paper: (1) reviews the history of the concept of secondary conditions as it applies to the health of individuals aging with long-term disabilities; (2) proposes a definition of secondary health conditions (SHCs) and a conceptual model for understanding the factors that are related to SHCs as individuals age with a disability; and (3) discusses the implications of the model for the assessment of SHCs and for developing interventions that minimize their frequency, severity and negative effects on the quality of life of individuals aging with $\mathrm{SCl}$ and other disabilities.

Methods: Key findings from research articles, reviews and book chapters addressing the concept of SHCs in individuals with $\mathrm{SCl}$ and other disabilities were summarized to inform the development of a conceptual approach for measuring SCl-related SHCs.

Conclusions: Terms used to describe health conditions secondary to $\mathrm{SCl}$ and other physical disabilities are used inconsistently throughout the literature. This inconsistency represents a barrier to improvement, measurement and for the development of effective interventions to reduce or prevent these health conditions and mitigate their effects on participation and quality of life. A working definition of the term SHCs is proposed for use in research with individuals aging with $\mathrm{SCl}$, with the goal of facilitating stronger evidence and increased knowledge upon which policy and practice can improve the health and well-being of individuals aging with a disability.

Spinal Cord (2012) 50, 373-378; doi:10.1038/sc.2011.150; published online 6 December 2011

\footnotetext{
Keywords: spinal cord injury; secondary conditions; secondary health conditions; comorbidities; aging with disability accelerated aging
}

\section{INTRODUCTION}

The importance of studying the acute and chronic health conditions that can develop as a result of having a long-term disability was first emphasized in the late $1980 \mathrm{~s},{ }^{1}$ perhaps due in part to increases in survivorship and longevity of the disabled population. Much of the research on this topic has relied on the term 'secondary conditions' to describe the consequences of long-term disability, without clear consensus on the definition of the term, or agreement on which health conditions are truly 'secondary' to disability. This lack of consistency, in conjunction with the absence of a model or framework for organizing the assessment and subsequent treatment of these health conditions, has impeded research in this area. The purpose of this paper is to facilitate progress in this area by: (1) reviewing the history of the 'secondary conditions' concept; (2) proposing a new label ('secondary health conditions (SHCs)') and definition of the concept; (3) presenting a model for understanding the relationships between these conditions and their precursors and sequelae; and (4) discussing the implications of the proposed model for future research.

\section{BACKGROUND AND HISTORY OF THE SECONDARY CONDITIONS CONCEPT}

It is now well-documented that individuals with long-standing physical disabilities are at a risk for the onset of a number of significant acute and/or chronic health conditions that may develop or be influenced by the presence of impairment and/or by the process of aging. ${ }^{2}$ The first conceptual paper to highlight the importance of systematic research into the nature, scope and impact of these health conditions was published in 1988 by Michael Marge. ${ }^{1}$ He used the term secondary disabilities to describe health conditions that develop after the onset of a primary disability. This definition was quite broad, and included all health conditions that emerged after the onset of the primary disability, whether or not they were related to the underlying

\footnotetext{
${ }^{1}$ Department of Rehabilitation Medicine, University of Washington School of Medicine, Harborview Medical Center, Seattle, WA, USA; ${ }^{2}$ National Rehabilitation Hospital, Washington, DC, USA; ${ }^{3}$ National Institute on Disability and Rehabilitation Research, Office of Special Education and Rehabilitative Services, U.S. Department of Education, Washington, DC, USA; ${ }^{4}$ Craig Hospital, Englewood, CO, USA; ${ }^{5}$ Department of Physical Medicine and Rehabilitation, University of Michigan, Ann Arbor, MI, USA and ${ }^{6}$ Medical University of South Carolina, Charleston, SC, USA

Correspondence: Professor MP Jensen, Department of Rehabilitation Medicine, University of Washington School of Medicine, Box 359612 , Harborview Medical Center, 325 Ninth Avenue, Seattle, WA 98104, USA.

E-mail: mjensen@uw.edu
}

Received 19 April 2011; revised 15 September 2011; accepted 17 September 2011; published online 6 December 2011 
impairment or to aging with a disability. One year later, Houk and Thacker ${ }^{3}$ used the term secondary complications to describe this same construct. Additional terms that have been used to label health conditions in individuals with disabilities include comorbid conditions or comorbidities, ${ }^{4,5}$ medical complications ${ }^{6,7}$ and associated conditions. ${ }^{8}$

In 1991, the Institute of Medicine (IOM) defined the term secondary conditions as ' ... any additional physical or mental health condition that occurs as a result of having a primary disabling condition' (italics added) and recommended more research to study their development and prevention. ${ }^{9}$ This resulted in the development and emergence of research programs to study the nature and scope of physical and psychological conditions that were thought to be the result of or influenced by a primary disability or by the long-term effects of aging with a disability. ${ }^{10,11}$

Since the 1991 IOM report, research focusing on the concept of health conditions following disability has significantly influenced the rehabilitation field by enhancing understanding of the associations between aging, or the duration of disability and important health outcomes in individuals with spinal cord injury (SCI) and other disabilities. SCI represents an excellent model for addressing this issue, given the multitude of SHCs that individuals with SCI have to contend with over their lifespan. Key findings from SCI research include the following:

(1) There is evidence for premature or accelerated aging for some organ systems in those with SCI compared with same-aged cohorts in the general population. ${ }^{12}$ This is particularly evident for the musculoskeletal, endocrine and cardiovascular systems. ${ }^{13-15}$

(2) The frequency of certain health conditions increases with duration of SCI. These include chronic pain, ${ }^{16}$ osteoporosis, ${ }^{15,17}$ pressure sores ${ }^{18}$ and kidney stones, ${ }^{18}$ among many others.

(3) Individual SHCs can be clustered into higher-order SHC constructs that may be useful for classifying and measuring these conditions in individuals with SCI, as well as other disabilities. ${ }^{19}$

Despite the acknowledged importance of the secondary conditions concept as it relates to the lives of individuals living with long-term disabilities, there is no clear agreed-upon definition of the concept to guide research. Thus, it is not surprising that there is a lack of consistency in the way that these conditions are conceptualized and measured in the scientific literature. Some scientists have expanded their definition of secondary conditions from the IOM's conceptualization to include disability-related social and activity limitations. ${ }^{4,8,20}$ In 2000, the Healthy People 2010 public health initiative was released, ${ }^{21}$ and expanded the types of conditions included under the term secondary conditions even further to include ' ... medical, social, emotional, mental, family or community problems that a person with a primary disabling condition likely experiences.' Clearly, a more consistent and limited definition is necessary to help organize and direct research efforts in rehabilitation and to improve measurement of the secondary conditions construct and enhance communication within other related fields, such as gerontology or geriatrics and public health.

Since March 2009, under the auspices of the National Institute on Disability and Rehabilitation Research (NIDRR), U.S, the Department of Education, a SCI Model Systems Special Interest Group on Aging (The SCI Model Systems Special Interest Group on Aging members include, in alphabetical order, Margaret Campbell (NIDRR liaison), Susan Charlifue, Anthony Chiodo, Martin Forchheimer, Suzanne L. Groah, Mark P. Jensen, James S. Krause, Dan Lammertse, Ivan R.
Molton, Laszlo Nagy, Denise Tate, and Jeanne Zanca.) has met regularly to discuss strategies to advance scientific understanding of issues related to aging with SCI. As an outgrowth of this process, the group recently developed a list of recommendations for future research on aging with $\mathrm{SCI}^{22}$ that includes the need for new information to improve rehabilitation interventions aimed at reducing the occurrence and impact of secondary conditions as people age with SCI. A necessary step in this process is to propose a clear definition of the secondary conditions concept that could facilitate the development of relevant measures as well as a working model for understanding the associations between SHCs and other important quality of life domains.

\section{PROPOSED DEFINITION AND CONCEPTUAL MODEL}

\section{Proposed definition}

We agree with the IOM's 1991 view that the secondary conditions concept should be limited to physical and psychological health conditions, ${ }^{5}$ and not include other domains that might be influenced by the presence of a disability, such as activity limitations or barriers to social participation. To help make this distinction clearer, we believe that it would be useful to include the word 'health' (that is, SHCs) when referring to the concept. As pointed out in the 2007 IOM report, The Future of Disability in America, distinguishing secondary physical and psychological health conditions from the other social and activity consequences that can result from a primary disability does not diminish the importance of the latter. ${ }^{23}$ Making this distinction facilitates improved measurement of the concept and allows us to develop models in which these individual domains can be viewed as mutually interactive, rather than separate independent dimensions within the same overarching construct of secondary conditions. For purposes of this paper, we have chosen the International Classification of Functioning, Disability and Health's (ICF) language and framework for describing and classifying health and health-related states because it provides a useful distinction between physical and psychological health conditions ('body functions and structures') and day-to-day functioning ('activities') and 'participation. ${ }^{24}$ Another reason for keeping with the ICF framework is that many investigators and policy makers in the fields of rehabilitation, public health and increasingly gerontology are familiar with and use the ICF language.

Any definition of SHCs should also acknowledge that these conditions can arise secondarily to a disability via at least two pathways. Having a disability may increase the risk of developing a health condition that (1) directly results from the impairment (for example, neurogenic bladder, spasticity) or (2) indirectly results because of factors related to the direct results of the impairment (for example, an increase in sedentary behaviors that contribute to the development of conditions such as obesity and diabetes, pressure ulcers that develop in part due to sensory impairment, urinary tract infections as a result of inadequate bladder emptying and reliance on urinary catheters). Through these pathways, living with SCI places individuals at higher risk of experiencing age-related chronic health conditions or comorbidities, such as osteoarthritis, hypertension and coronary artery disease, with greater severity and at a younger age than their non-disabled counterparts. This process contributes to what has been described as 'accelerated aging.' ${ }^{12}$ Any definition of SHCs should acknowledge these two types of influences on the health status of individuals with SCI in order to facilitate their measurement and study. Although our specific focus is on SCI, we propose a general definition of SC that is applicable to individuals with a range of physical disabilities.

On the basis of the above considerations, we define SHCs as 'physical or psychological health conditions that are influenced 
directly or indirectly by the presence of a disability or underlying physical impairment.' Although this definition implies causality, it would be too limiting to restrict it to simply linear causal relationships. Rather, one must take a multi-dimensional view of disability and health, such that a change in one health condition may well influence the presence or severity of other conditions.

It is also useful to examine and apply some previously established criteria for causation to help determine whether or not a condition is influenced by a disability, ${ }^{25,26}$ with three of these criteria being particularly relevant for determining that a health condition is or is not secondary to a disability. They are (1) temporality-the causal agent precedes the SHC or worsening of the condition; (2) biological gradient-more of a potentially causal agent leads to an increase in the frequency or severity of the condition; and (3) plausibility/coherence-a causal relationship is biologically or psychologically reasonable, given the state of our scientific knowledge.

Temporality may be established through diagnostic interview. One strategy that may be used to meet the biological gradient criterion is to administer the measures of potential SHCs to samples of individuals with disabilities, and then compare the rates of those conditions between (1) participants who have had the disability for a relatively long time and (2) a similar-aged subgroup with relatively recent onset disability. This design controls for age at onset and chronological age effects, so differences in the frequency of conditions between the two groups potentially could be due to disability duration-with the caveat that the inception era cohort effect (that is, the health care available at the time of disability onset) is a potential confounder. Two such studies support urinary tract infections, kidney stones, fractures, contractures, curvature of the spine and pressure sores as meeting the biological gradient criterion for being SHCs in individuals with SCI. ${ }^{18,27}$

The plausibility/coherence criterion is also addressed in our working definition. It makes sense, based on what is known about biology, that health conditions associated with a sedentary lifestyle (for example, higher fat-mass levels, diabetes, cardiovascular conditions and bone-density loss) would be influenced by having a disability. This plausibility, when combined with evidence that these conditions occur more frequently in individuals with SCI than in non-disabled individuals, supports them as SHCs. ${ }^{28,29}$ Although a systematic review of the research literature to identify all health conditions that meet our proposed criteria is beyond the scope of this paper, some common examples of these are listed in Table 1.

\section{Moving forward: models for assessing and understanding SHCs} Although there is much that we have learned about SHCs, scientific understanding of the construct and how to intervene to reduce the potential negative effects of these conditiins will be further facilitated by (1) a measurement model for assessing SHCs and (2) a conceptual framework both for formulating hypotheses regarding the causes and effects of these conditions and for developing interventions that will reduce their frequency, severity and negative impact.

Measurement. models of SHCs. As mentioned above, the potential pool of possible SHCs in our definition consists of the ICF's list of body functions and structures. ${ }^{24}$ However, our understanding of the specific secondary conditions that meet the criteria for SHCs, as well as the factor structure of measures of these conditions, is limited at this point. On the basis of a review of the literature, we have identified a preliminary list of SHCs for people with SCI (Table 1) that meet the above criteria. Further work should focus on developing, testing and
Table 1 A list of secondary health conditions related to $\mathrm{SCl}$ and associated citations

Secondary health condition

Cardiovascular disease 38,39

Chronic pain 40

Constipation 41

Contractures $^{27}$

Deep vein thrombosis/pulmonary embolism 42

Depression $^{42}$

Fractures $^{27}$

Heterotopic ossification 43

Imobilization hypercalcemia ${ }^{44}$

Insomnia/sleep difficulties ${ }^{37}$

Kidney stones ${ }^{18,27}$

Neurogenic bladder 45

Neurogenic bowel ${ }^{46}$

Non-urinary tract infections ${ }^{27}$

Obesity $^{29}$

Osteoporosis/Bone density loss $15,17,29$

Pressure sores ${ }^{18,41}$

Restrictive lung disease ${ }^{47}$

Septicemia $^{41}$

Sexual dysfunction 48,49

Sleep apnea 50

Spasticity 51

Urinary tract infection ${ }^{52}$

Abbreviation: $\mathrm{SCl}$, spinal cord injury.

refining validated measures of these SHCs, for use in research with SCI and ultimately with other disabilities.

We anticipate that the health conditions that meet the criteria for being 'secondary' to a disability (that is, temporality, biological gradient and plausibility criteria) very likely cluster into subdomains. A recent study using a factor analysis of selected health outcome measures yielded six health-outcome factors, supporting this possibility. 19 Three of the factors that emerged were clearly healthcondition factors reflecting (1) pressure sores, (2) illness/infections and (3) orthopedic conditions. In addition, a measure of emotional functioning (depression) was associated with a separate factor, providing support for a distinction between psychological and physical SHCs.

An example of the type of applicable measure as relates to SCI is the Spinal Cord Injury Secondary Conditions Scale (SCI-SCS), which is based on the Secondary Conditions scale, ${ }^{11}$ and assesses 16 conditions in terms of how they limit activity. ${ }^{30}$ As we learn more about specific conditions that are influenced by certain disabilities, measures such as the SCI-SCS will become increasingly useful. Furthermore, the recently developed International SCI Standards and Datasets includes several of these conditions, and provides a framework for the development of new standardized measures that best capture the concept of SHCs. ${ }^{31-34}$

Conceptual models of SHCs. We propose a framework that could be used as a point of departure for understanding the predictors and effects of SHCs in Figure 1. This model hypothesizes that both disability factors, such as the presence of impairment or significant restrictions in activity or participation, as well as age-related factors (such as chronological age, age at disability onset, disability inception era and duration of disability), can have direct effects on the development and trajectory of SHCs. The model also hypothesizes that disability and age-related variables can influence the effects of 


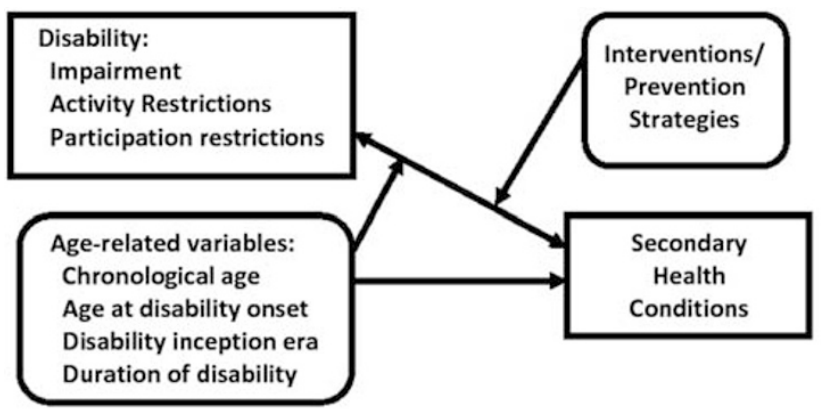

Figure 1 Proposed model of the effects of disability on SHCs, and how this relationship can be influenced by both age-related variables and intervention/prevention strategies.

disability on SHCs (for example, via the 'accelerated aging' pathway noted to occur in individuals with physical disabilities ${ }^{4,35-36}$ ). SHCs, in turn, can also influence disability.

The model also explicitly notes that interventions and adaptive coping responses can influence both the development of SHCs and their negative effects on activity and participation. The interventions that can be envisioned to potentially influence the development or course of SHCs target many of the (changeable) personal factors that are included in the ICF model of disability, such as fitness, lifestyle, health habits, individual psychological resources and coping style. ${ }^{24}$

Need for improved analytic strategies. Research is still needed to thoroughly identify and evaluate the factors that influence and contribute to the development of SHCs. For example, although we know that aging influences the musculoskeletal system in individuals with SCI differently than it does in individuals who are not disabled, ${ }^{35}$ we do not know yet how neuromuscular aging affects respiratory complications in older adults with SCI. Similarly, the lack of evidence on natural aging processes of the gastrointestinal and genitourinary systems after SCI indicates there is not enough evidence to conclude that aging itself influences these body systems differently in individuals with SCI than in individuals without SCI. ${ }^{13}$ Also, there are a large number of behavioral and life-style factors that influence the development and course of SHCs. For instance, although the risk of pressure ulcers clearly increases dramatically at SCI onset, behavioral factors have a role in their development and course. ${ }^{16}$ These behavioral factors can, in turn, be influenced by treatments that buffer the potential negative effects that other factors (such as age) have on secondary conditions (via intervention/prevention strategies, as indicated in Figure 1).

Although a measurement model will help guide the selection of variables to assess, and ultimately may help us determine how best to combine these variables into composite factors, a conceptual model provides guidance regarding both (1) hypotheses to test and (2) analytic approaches needed to better understand the factors that impact the development and effects of SHCs. As such, our proposed model (see Figure 1) indicates that in order to design a cross-sectional study to determine what factors may predict pressure sores, it would be important to assess chronological age, duration of SCI, age at SCI onset, date of SCI (as a measure of inception era), the prevention strategies used to influence skin health (for example, frequency of position or weight shifts, type of seating surface), and both the frequency and severity of pressure sores. Our conceptual model predicts that one or more of the age-related variables (chronological age, age at injury, inception era cohort, duration of injury ${ }^{37}$ ) as well as prevention strategies could interact with the disability to predict the frequency and development of pressure sores. Importantly, the model also predicts that the effects of disability and age-related variables on activities and participation are mediated, at least in part, by their effects on SHCs.

Understanding the importance of age-related variables in the development of SHCs could also be furthered by more longitudinal research. Such research could track the trajectories of SHCs in people aging with SCI, relative to matched individuals without SCI. Finally, the importance of prevention strategies for enhancing health in the general population has been examined in multiple studies, but such research in SCI is limited. Identifying and studying the effects of these prevention strategies represents a critical area of research focus.

\section{SOME LIMITATIONS OF THE SHCS CONCEPT}

Despite the clear importance of clarifying and understanding the concept of SHCs, as well as their precursors, consequences, and potential interventions, the study and terminology of SHCs does have a number of limitations that should be acknowledged. First, although many of these conditions are pertinent across disabilities, others are disability-specific. Because of this, any definition that stipulates a standardized set of conditions will not likely be useful in cross-disability research. Moreover, exclusive reliance of the label SHCs by rehabilitation researchers and practitioners has the potential to create unnecessary divisions in the study of chronic health conditions among disabled, non-disabled and aging populations. For example, urinary tract infections are a significant and common secondary health condition for many individuals with SCI. But interventions developed by SCI researchers to better manage this SHC may also be useful for clinicians who serve non-disabled individuals with neurogenic bladder needs, among whom UTIs are not referred to as 'SHCs.' Similarly, general research on UTIs in nonSCI and aging populations would likely be of interest to SCI clinicians and researchers. Rigid terminological differences can potentially serve as a barrier to the widespread exchange of this information. Thinking exclusively in terms of SHCs, as opposed to the more general concept of chronic health conditions, has the potential to restrict important cross-fertilization among researchers from different fields, thereby limiting the benefits that would occur from shared terminology and more active communication. The solution to this limitation may be to not discard the concept of SHCs altogether, but rather to ensure that disability researchers do not focus exclusively on the disability and rehabilitation literature.

It is also important to remember that individual consumers may not make clear distinctions regarding whether their current health conditions are or are not influenced by their disabilities. They may even find this issue irrelevant. For example, if they have heart disease, they require adequate evaluation and treatment of their heart disease, and may find the discussion of whether or not this chronic condition is disability-related to be invalidating. At the same time, it is also important to understand that many consumers may not be aware of the influence of their disability on a health problem or its treatment. It is critical that we understand these influences so we can provide guidance to our patients in order to improve health and longevity and prevent further disability or activity restrictions. From a point of view of costs and successful health-care models, prevention has a significant role in terms of identifying those at risk for developing such conditions and delivering early treatments to minimize negative effects.

\section{CONCLUDING COMMENTS}

Over the past 50 years, the field of medical rehabilitation has made incredible strides in identifying and treating injuries and illnesses that 
cause disability. With increases in longevity across disability groups, the field is necessarily shifting its focus to the management of health conditions that develop as individuals' age with disability. Research efforts can have a significant impact in terms of understanding and treating these conditions, and disability researchers are presented with a unique opportunity to influence the quality of life of individuals living with disabilities such as SCI. Moreover, the findings of rehabilitation researchers in this area will likely be very useful to researchers who study aging in other populations (and vice versa), and the resulting cross-fertilization among disciplines could have many benefits on our knowledge base, and ultimately on the health and wellness of aging individuals, regardless of disability status. Although rigorous research is not entirely dependent on a clear consensus of key terms, such as consensus ensures better communication of results, maximizes efficiency by limiting unintentional replication of previous studies, allows for more effective meta-analysis and generally moves the field forward. We have proposed one definition for the term SHCs as well as a conceptual model that we hope will help facilitate and guide research in this area.

\section{DATA ARCHIVING}

There was no data to deposit.

\section{CONFLICT OF INTEREST}

This paper is intended to promote the exchange of ideas among researchers and policymakers. The views expressed in it are solely those of the authors and do not necessarily reflect the views or position of the U.S. Department of Education or the National Institute on Disability and Rehabilitation Research.

\section{ACKNOWLEDGEMENTS}

Work on this manuscript was supported, in part, by grants to the Model Spinal Cord Injury Systems (H133N060028, H133N060005, H133N060032, and H133N060009) and the Rehabilitation Research and Training Center on Aging with Physical Disabilities (H133B080024) from the National Institute on Disability and Rehabilitation Research (in the Office of Special Education and Rehabilitation Services, U.S. Department of Education), and was endorsed by the MSCIS Special Interest Group on Aging with SCI.

1 Marge M. Health promotion for persons with disabilities: moving beyond rehabilitation. Am J Health Promot 1988; 2: 29-35.

2 Halstead LS, Rossi CD. New problems in old polio patients: results of a survey of 539 polio survivors. Orthopedics 1985; 8: 845-850.

3 Houk VN, Thacker SB. The Centers for Disease Control program to prevent primary and secondary disabilities in the United States. Public Health Rep 1989; 104: 226-231.

4 Campbell ML, Sheets D, Strong PS. Secondary health conditions among middle-aged individuals with chronic physical disabilities: implications for unmet needs for services. Assistive Technol 1999; 11: 105-122.

5 Turk M. Secondary conditions and disability. In: Field MJ, Jette AM, Martin L (eds). Workshop on disability in America. A new look. Summary and background papers. Board on Health Sciences Policy, Institute of Medicine of the National Academies, The National Academies Press: Washington DC, 2006, pp. 185-193.

6 Cosar SN, Yemisci OU, Oztop P, Cetin N, Sarifakioglu B, Yalbuzdag SA et al. Demographic characteristics after traumatic and non-traumatic spinal cord injury: a retrospective comparison study. Spinal Cord 2010; 48: 862-866.

7 Divanoglou A, Westgren N, Bjelak S, Levi R. Medical conditions and outcomes at 1 year after acute traumatic spinal cord injury in a Greek and a Swedish region: a prospective, population-based study. Spinal Cord 2010; 48: 470-476.

8 Turk MA, Overeynder JC, Janicki MP (eds) Uncertain future-Aging and cerebral palsy: Clinical concerns. Albany, NY: New York State Developmental Disabilities Planning Council, 1995.

9 Pope AM, Tarlov AR. (eds) Disability in America: Toward a national agenda for prevention. National Academy Press: Washington, DC, 1991.

10 Maynard FM, Julius M, Kirsch N, Lampman R, Peterson C, Tate D et al. The late effects of polio: A model for identification and assessment of preventable secondary disabilities. Final Report to Centers for Disease Control (\#U59/CCU5:0338-03).
Department of Physical Medicine and Rehabilitation, University of Michigan: Ann Arbor, MI, 1991.

11 Seekins T, Clay J, Ravesloot C. A descriptive study of secondary conditions reported by a population of adults with physical disabilities served by three independent living centers in a rural state. J Rehabil 1994; 60: 47-51.

12 Kemp BJ, Mosqueda L (eds) Aging with a disability: What the clinician needs to know. The Johns Hopkins University Press: Baltimore, MD, 2004.

13 Hitzig SL, Campbell KA, McGillivray CF, Boschen KA, Craven BC. Understanding age effects associated with changes in secondary health conditions in a Canadian spinal cord injury cohort. Spinal Cord 2010; 48: 330-335.

14 Spungen AM, Adkins RH, Stewart CA, Wang J, Pierson Jr RN, Waters RL et al. Factors influencing body composition in persons with spinal cord injury: a cross-sectional study. J Appl Physiol 2003; 95: 2398-2407.

15 Bauman WA, Spungen AM. Body composition in aging: Adverse changes in ablebodied persons and in those with spinal cord injury. Top Spinal Cord Inj Rehabil 2001; 6: 22-36.

16 Gelis A, Dupeyron A, Legros P, Benaim C, Pelissier J, Fattal C. Pressure ulcer risk factors in persons with spinal cord injury part 2: the chronic stage. Spinal Cord 2009; 47: 651-661.

17 Jiang SD, Dai LY, Jiang LS. Osteoporosis after spinal cord injury. Osteoporos Int 2006; 17: 180-192.

18 McGlinchey-Berroth R, Morrow L, Ahlquist M, Sarkarati M, Minaker KL. Late-life spinal cord injury and aging with a long term injury: characteristics of two emerging populations. J Spinal Cord Med 1995; 18: 183-193.

19 Krause JS, Reed KS, McArdle JJ. A structural analysis of health outcomes after spinal cord injury. J Spinal Cord Med 2010; 33: 22-32.

20 Simeonsson RJ, Leskinen M. Disability, secondary conditions and quality of life: Conceptual issues. In: Simeonsson RJ, McDevitt LN, eds. Issues in disability and health: The role of secondary conditions and quality of life. University of North Carolina Press: Chapel Hill, NC, 1999, pp 51-72.

21 Healthy People 2010. Secretary's Advisory Committee on Health Promotion and Disease Prevention Objectives for 2010. Available at: http://www.healthypeople.gov/ 2020 Accessed 2/15/2011.

22 Groah SL, Charlifue S, Tate D, Jensen MP, Molton IR, Forchheimer M et al. Spinal cord injury and aging: challenges and recommendations for future research. Am J Phys Med Rehabil (e-pub ahead of print 15 June 2011).

23 Field MJ, Jette AM eds. The Future of Disability in America. National Academic Press: Washington, DC, 2007

24 World Health Organization. International classification of functioning, disability and health: ICF. World Health Organization: Geneva, 2001.

25 Hill AB. The environment and disease: association or causation? Proc $R$ Soc Med 1965 58: 295-300.

26 Rothman KJ, Greenland S. Causation and causal inference in epidemiology. Am J Public Health 2005; 95(Suppl 1): S144-S150.

27 Krause JS. Aging after spinal cord injury: an exploratory study. Spinal Cord 2000; 38: 77-83.

28 Banerjea R, Sambamoorthi U, Weaver F, Maney M, Pogach LM, Findley T. Risk of stroke, heart attack, and diabetes complications among veterans with spinal cord injury. Arch Phys Med Rehabil 2008; 89: 1448-1453.

29 Spungen AM, Wang J, Pierson Jr RN, Bauman WA. Soft tissue body composition differences in monozygotic twins discordant for spinal cord injury. J App/ Physiol 2000; 88: 1310-1315.

30 Kalpakjian CZ, Scelza WM, Forchheimer MB, Toussaint LL. Preliminary reliability and validity of a Spinal Cord Injury Secondary Conditions Scale. J Spinal Cord Med 2007; 30: 131-139.

31 Widerstrom-Noga E, Biering-Sorensen F, Bryce T, Cardenas DD, Finnerup NB, Jensen MP et al. International spinal cord injury pain basic data set. Spinal Cord 2008; 46: 818-823.

32 Krassioukov A, Alexander MS, Karlsson AK, Donovan W, Mathias CJ, Biering-Sorensen F. International spinal cord injury cardiovascular function basic data set. Spinal Cord 2010; 48: 586-590.

33 Krogh K, Perkash I, Stiens SA, Biering-Sorensen F. International bowel function basic spinal cord injury data set. Spinal Cord 2009; 47: 230-234.

34 Biering-Sorensen $F$, Craggs M, Kennelly M, Schick E, Wyndaele JJ. International urodynamic basic spinal cord injury data set. Spinal Cord 2008; 46: 513-516.

35 Hitzig SL, Eng JJ, Miller WC, Sakakibara BM. An evidence-based review of aging of the body systems following spinal cord injury. Spinal Cord 2011; 56: 15-25.

36 Bauman WA, Spungen AM. Disorders of carbohydrate and lipid metabolism in veterans with paraplegia or quadriplegia: a model of premature aging. Metabolism: clinical and experimental 1994; 43: 749-756.

37 Jensen MP, Hirsh AT, Molton IR, Bamer AM. Sleep problems in individuals with spinal cord injury: frequency and age effects. Rehabil Psychol 2009; 54: 323-331.

38 Groah SL, Weitzenkamp D, Sett P, Soni B, Savic G. The relationship between neurological level of injury and symptomatic cardiovascular disease risk in the aging spinal injured. Spinal Cord 2001; 39: 310-317.

39 Garshick E, Kelley A, Cohen SA, Garrison A, Tun CG, Gagnon D et al. A prospective assessment of mortality in chronic spinal cord injury. Spinal Cord 2005; 43(7): 408-416.

40 Jensen MP, Hoffman AJ, Cardenas DD. Chronic pain in individuals with spinal cord injury: a survey and longitudinal study. Spinal Cord 2005; 43: 704-712.

41 Valles M, Vidal J, Clave P, Mearin F. Bowel dysfunction in patients with motor complete spinal cord injury: clinical, neurological, and pathophysiological associations. $A m$ J Gastroenterol 2006; 101: 2290-2299. 
42 Dryden DM, Saunders LD, Rowe BH, May LA, Yiannakoulias N, Svenson LW et al. Utilization of health services following spinal cord injury: a 6 -year follow-up study. Spinal Cord 2004; 42: 513-525.

43 van Kuijk AA, Geurts AC, van Kuppevelt HJ. Neurogenic heterotopic ossification in spinal cord injury. Spinal Cord 2002; 40: 313-326.

44 Meythaler JM, Tuel SM, Cross LL. Successful treatment of immobilization hypercalcemia using calcitonin and etidronate. Arch Phys Med Rehabil 1993; 74 316-319.

45 Jeong SJ, Cho SY, Oh SJ. Spinal cord/brain injury and the neurogenic bladder. Urol Clin North Am 2010; 37: 537-546.

46 Liu CW, Huang CC, Chen CH, Yang YH, Chen TW, Huang MH. Prediction of severe neurogenic bowel dysfunction in persons with spinal cord injury. Spinal Cord 2010; 48: 554-559.
47 Spungen AM, Grimm DR, Schilero G, Lenner R, Oei E, Bauman WA et al. Relationship of respiratory symptoms with smoking status and pulmonary function in chronic spinal cord injury. J Spinal Cord Med 2002; 25: 23-27.

48 Lombardi G, Mondaini N, Macchiarella A, Del Popolo G. Female sexual dysfunction and hormonal status in spinal cord injured (SCI) patients. J Androl 2007; 28: 722-726.

49 Brown DJ, Hill ST, Baker HW. Male fertility and sexual function after spinal cord injury. Prog Brain Res 2006; 152: 427-439.

50 Biering-Sorensen $F$, Jennum P, Laub M. Sleep disordered breathing following spinal cord injury. Respir Physiol Neurobiol 2009; 169: 165-170.

51 Adams MM, Hicks AL. Spasticity after spinal cord injury. Spinal Cord 2005; 43 : 577-586.

52 Foxman B, Brown P. Epidemiology of urinary tract infections: transmission and risk factors, incidence, and costs. Infect Dis Clin North Am 2003; 17: 227-241. 